\title{
DIVISION 32 DIRECTORY
}

\section{President}

Alvin R. Mahrer

School of Psychology

University of Ottawa

Ottawa, Canada K1N 6N5

President-Elect

Harold Greenwald

5135 Pacifica Drive

San Diego, CA 92109

Treasurer

Constance L. Moerman

7111 Braeburn Place

Bethesda, MD 20817

Members-at-Large

Arnold Bernstein

26 W. Ninth Street

New York, NY 10011

Amedeo Giorgi

Psychology Department

Duquesne University

Pittsburgh, PA 15282

Gloria B. Gottsegen

Dept. of Specialized Services

H. Lehman College-CUNY

Bronx, NY 10468

Susan J. Herman

1296 Oxford Lane

Union, NJ 07083

John Tisdale

Cedar Crest College

Allentown, PA 18104

Nora Weckler

1234 Rochedale Lane

Los Angeles, CA 90049
Past President

M. Brewster Smith

Stevenson College

University of California

Santa Cruz, CA 95064

Secretary

Mary Jo Meadow

1204 Highland Avenue

Mankato, MN 56001

APA Council of Representatives

Carmi Harari

Humanistic Psychology Center of New York

473 West End Avenue

New York, NY 10024

Bulletin Editor

Christopher M. Aanstoos

Psychology Department

West Georgia College

Carrollton, GA 30118

Membership Chair

Frederick J. Wertz

Division of Social Science

Fordham University, Lincoln Center

New York, NY 10023

Program Chair

Adrianne Aron

1807 Vine Street

Berkeley, CA 94703

Hospitality Suite Chair

John M. Schmidt

6116 Montrose Road

Cheverly, MD 20785 